\title{
Connectivity Model for Wireless Mesh Networks
}

\author{
Mohamed Abou El Saoud, Hussein Al-Zubaidy, SamyMahmoud, \\ Department of Systems and Computer Engineering, Carleton University \\ Email: \{moaz, mahmoud, hussein\}@ sce.carleton.ca
}

\begin{abstract}
This paper presents a novel methodology for finding the network connectivity in wireless mesh networks while taking into account dependencies existing between links of geometrically co-located nodes, as well as the effect of a finite network boundary. We show that the commonly used assumption of link independence almost always underestimate the network connectivity. We also show that the assumption of infinite network boundary is invalid as it overestimates the network connectivity by a non negligible amount. We use our methodology to derive accurate upper bounds for network connectivity in the optimal triangular lattice topology. A comparison study shows that the error due to either assumptions depends on the link connectivity as well as the network size, and can be very significant. The devised methodology can also be applied to any lattice topology in order to quantify the error and define the range of link conductivities within which the assumptions can be used.
\end{abstract}

\section{INTRODUCTION}

Network Connectivity is a vital metric that is frequently utilized in the design and network planning process, as well as in describing and comparing networks. Network connectivity often refers to the probability that nodes in a network can communicate with each other at any given time.

In wireless networks, connectivity is often expressed in terms of individual links' connectivities. To the best of our knowledge, all previous work embeds either or both of the following assumptions:

1) Inter-node links' connectivities are independent of one another.

2) Border nodes are assumed to have the same connectivity as the interior nodes in a network.

The first assumption implies that the probability a node is connected is independent of that of the other nodes in the same network. The second assumption is based on the invalid perception that if the network is large enough to be assumed infinite, then the border nodes will have the same connectivity as the rest of the interior nodes in the network.

Although these simplifications are adopted by many researchers such as [1]-[5], the impacts of such assumptions were never examined.

In this work, we present a methodology to derive a closed form upper bound for the node connectivity in wireless networks taking into account dependencies existing between links of geometrically co-located nodes, as well as the effect of a finite network boundary. Although we derive the model and analyze the results for the optimal triangular lattice topology, the qualitative conclusions will generally hold for similar topologies.
Next in Section II, we define the problem. Section III presents the derivation of the model. We present our results in Section IV. The conclusions are given in Section V.

\section{Problem Definition}

A network formed by a set of nodes located in an area $\mathbf{A}$ is said to be fully connected if and only if there is a path between each pair of nodes in A. For a path to exist between a pair of wireless nodes, all links along the path must be connected. Hence, in wireless networks, connectivity is usually expressed in terms of the probability of individual link connectivity.

In radio links, the probability that a link exist between two nodes depend among other things on the geometric distance between the two nodes. Hence, link connectivity in one direction implicitly depends on that of the opposite direction. The amount of correlation, or the extent of dependency between the two unidirectional links is affected by several factors such as multi-path fading, shadowing, and the difference in the nodes' transmitting powers.

In this work, we present a methodology for finding a closed form expression for the connectivity in wireless networks while taking into account inter-node links dependencies.

A wireless network is composed of nodes that are allocated in an area $\mathbf{A}$ according to the topology $\mathbf{T}$. Define the following:

- $\mathbb{L}$ to be the set of wireless nodes in $\mathbf{A} . \mathbb{L}=\{1,2, \ldots, L\}$.

- $\mathbb{D}_{i} \subset \mathbb{L}$ to be the set of nodes that are neighbors of node $i \in \mathbb{L}$, that is, nodes that are one-hop away from node $i$.

- $l_{i j}$ to be the event that a link exists between node $i$ and node $j$, where $i \in \mathbb{L}$ and $j \in \mathbb{D}_{i}$.

- $r_{i j}$ to be the probability that a link exists between node $i$ and node $j$, that is $P\left(l_{i j}\right), \forall i \in \mathbb{L}$ and $\forall j \in \mathbb{D}_{i}$, where $P(e)$ is the probability of event $e$ happening.

\section{A. Basic Assumptions}

Some realistic assumptions were made, these are:

- Similar to most researchers, we consider interference as a capacity-affecting factor that reduces the capacity of a link instead of lowering the probability of connectivity.

- Symmetric bidirectional links that are fully dependant. That is, $P\left(l_{i j}\right)=P\left(l_{j i}\right) \forall i \in \mathbb{L}$ and $\forall j \in \mathbb{D}_{i}$,

- Nodes are equidistantly located, and that $\mathbf{A}$ has a homogenous shadowing and fading conditions. Hence, $P\left(l_{i j}\right)=$ $r_{i j}=r, \forall i \in \mathbb{L}$ and $\forall j \in \mathbb{D}_{i}$.

- $P\left(l_{i j}\right)=0 \quad \forall j \notin \mathbb{D}_{i}$, i.e. node $i$ can only be connected through one or more of its neighbors (i.e. nodes in $\mathrm{D}_{i}$ ). 


\section{B. Upper bound for connectivity}

Finding the exact connectivity of a wireless network is very costly in terms of computational complexity. Instead, we find the probability that none of the nodes in the network is isolated, $P$ (no node isolated). However, a situation may arise where one or more islands of nodes are formed causing the network to be partitioned. Hence, the probability that none of the nodes in a network is isolated is an upper bound for the connectivity of a network, that is:

$$
\begin{aligned}
P(\text { network connected }) & \leq P(\text { no node } \text { isolated }) \\
& =P\left(n_{1}, n_{2}, \ldots, n_{L}\right)
\end{aligned}
$$

where $n_{i}$ is a random variable that denotes the connectivity of node $i$, that is:

$$
n_{i}= \begin{cases}1 & \text { if node } i \text { is not isolated with probability } P\left(n_{i}\right) \\ 0 & \text { if node } i \text { is isolated with probability } 1-P\left(n_{i}\right)\end{cases}
$$

where

$P\left(n_{i}\right) \equiv P\left(\right.$ at least one link $\left(l_{i j}: \forall j \in \mathbb{D}_{i}\right)$ is connected $)$

According to Erdös and Rényi [6], this bound for connectivity is tight for nodes whose topology are purely random. Hence, this upper bound is expected to be even tighter for a network of fixed topology, and where nodes are placed equidistantly apart.

\section{Link and Node Independence}

We compare our computed upper bound for connectivity, $P($ no node isolated $)$, that takes into account link dependance and a finite network boundary, with the upper bound, $P(\text { no node isolated })^{*}$ commonly utilized in the literature, and which assumes link independence. The probability that none of the nodes in a given network are isolated assuming independent links is:

$$
\begin{aligned}
P(\text { no node isolated })^{*} & =\prod_{\forall i \in \mathbb{L}} P\left(n_{i}\right) \\
& =\prod_{\forall i \in \mathbb{L}}\left(1-P\left(\overline{n_{i}}\right)\right) \\
& =\prod_{\forall i \in \mathbb{L}}\left(1-\prod_{\forall j \in \mathbb{D}_{i}} P\left(\overline{l_{i j}}\right)\right)
\end{aligned}
$$

where $\bar{\alpha}$ denotes the complement of event $\alpha$; the first line follows from applying the assumption of node independence on (1); the second line follows the complement rule; the third line follows from applying the assumption of link independence and the complement rule on (3).

\section{Infinite Boundary Assumption}

Prior to this work, most researchers avoid the effect of reduced node connectivity of border nodes by assuming that the networks examined are infinitely large. Border nodes have less neighbors, and hence, less chances of being connected.
The embedded assumption here is that the effect of such nodes on the overall network connectivity in an infinitely large network is negligible. We argue against that, and derive expressions for network connectivity that take into account the reduced connectivity of border nodes.

\section{Connectivity Model}

\section{A. Modeling Link Dependencies}

We apply our methodology to find the connectivity in a wireless network, whose topology, $\mathbf{T}$, is a triangular lattice. This topology is obtained when nodes are placed on the vertices of an equilateral triangular lattice, or equivalently, at the centers of regular hexagons. A 2-tier triangular lattice topology is shown in Figure 1. It was previously proven that the triangular lattice topology results in the minimum number of nodes needed to achieve full coverage of a plane [7]. The goal is to derive the joint probability in (1) for this network.

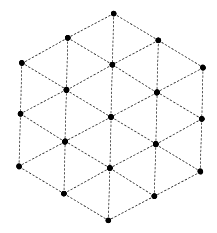

Fig. 1: Nodes in a Triangular Lattice Topology

Define $\mathbb{L}_{i} \subset \mathbb{L}$ to be the set of indices for the nodes that have not yet been considered. That is:

$$
\mathbb{L}_{i}=\{i+1, i+2, \ldots, L\}
$$

where the nodes in $\mathbf{T}$ are numbered in sequence from the center outwards, starting with node 1 for the center node.

Define the set $\mathbb{Q}_{i} \subseteq \mathbb{D}_{i}$ to be the set of all neighboring nodes of $i$ that have not yet been considered. Therefore,

$$
\mathbb{Q}_{i}=\mathbb{L}_{i} \cap \mathbb{D}_{i} \text {. }
$$

Define $q_{\xi}^{i} \in \mathbb{Q}_{i}$ to indicate the $\xi$ th element in $\mathbb{Q}_{i}$, and $N \equiv$ $\left|\mathbb{Q}_{i}\right|$. Hence, we can re-write (1) as follows:

$$
\begin{aligned}
P & \left(n_{1}, n_{2}, \ldots, n_{L}\right) \\
& =P\left(n_{1} \mid n_{2}, \ldots, n_{L}\right) \cdot P\left(n_{2}, \ldots, n_{L}\right) \\
& =\prod_{i=1}^{L-1} P\left(n_{i} \mid n_{i+1}, \ldots, n_{L}\right) \cdot P\left(n_{L}\right) \\
& =\prod_{i=1}^{L-1} P\left(n_{i} \mid n_{q_{1}^{i}}, \ldots n_{q_{N}^{i}}\right) \cdot P\left(n_{L}\right)
\end{aligned}
$$

where the first line follows from the definition of conditional probability; the second line is obtained by iteratively applying the definition of conditional probability; the third line follows from the assumption that node $i$ can only be connected through one or more of its neighbors, that is, $n_{i}$ depends only on nodes in $\mathbb{Q}_{i}$.

Furthermore,

$$
P\left(n_{i} \mid n_{q_{1}^{i}}, \ldots n_{q_{N}^{i}}\right)=\frac{P\left(n_{i}, n_{q_{1}^{i}}, \ldots n_{q_{N}^{i}}\right)}{P\left(n_{q_{1}^{i}}, \ldots n_{q_{N}^{i}}\right)} \quad \text { for } i<L
$$


also using the definition of conditional probability.

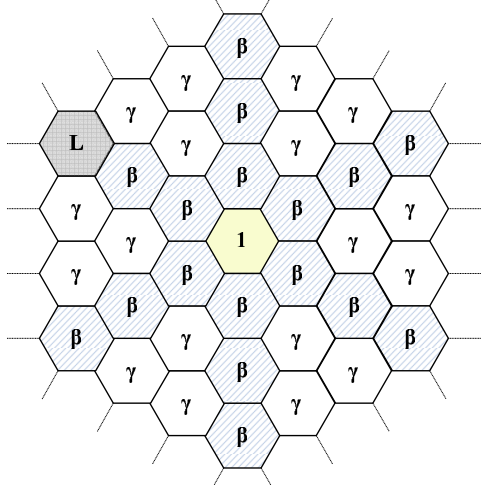

Fig. 2: Location of various node types

Depending on the location of node $i$ in the topology, the number of elements in the set $\mathbb{Q}_{i}$ will be different. Therefore, the quotient resulting from (6) will differ depending on the location of node $i$. Because of the assumption of equal network-wide link connectivity, only the number of elements in $\mathbb{Q}_{i}$ matters. The exact node id is irrelevant. In order to simplify matters, we leverage on the geometry and recursive decomposition of the triangular lattice topology. Similar to most research, we also ignore the border effect of the outer most tier (nodes in the outer tier only have three or four neighbors); in a way, we assume that the network exists in an infinitely large topology, so every node has 6 neighbors. Hence, there are four cases to consider (Refer to Figure 2):
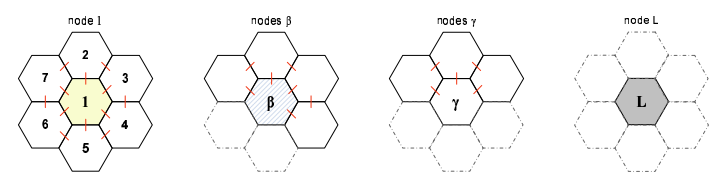

Fig. 3: Neighbors and Dependencies for various node types

Case 1: node 1

We start by first considering the node located at the center of the topology, that is node $1 . \mathrm{D}_{1}$ similar to $\mathrm{D}_{i}$ for all nodes $i$ will be comprised of the six nodes surrounding node $i$. Since node 1 is the first node to consider in the computation, $\mathbb{L}_{1}$ will contain all nodes in the topology except this center node. Hence, $\mathbb{Q}_{1}=\mathbb{L}_{1} \cap \mathbb{D}_{1}=\mathbb{D}_{1}$. That is, $\mathbb{Q}_{1}$ is comprised of all six nodes neighboring node 1 , and $\left|\mathbb{Q}_{1}\right|=6$. There are 12 link dependencies as denoted by the dashes in Figure 3.

Case 2: nodes of type $\beta$

We find that for each node of type $\beta, \mathbb{Q}_{i}$ is comprised of four of the six nodes neighboring it, that is $\left|\mathbb{Q}_{i}\right|=4$. The two remaining nodes are not elements of $\mathbb{L}_{i}$ (they were already considered in the previous term and hence eliminated from the consequent terms), and therefore are not elements in $\mathbb{Q}_{i}$. There are 7 link dependencies as denoted by the dashes in Figure 3. We also note that except for the outer tier, each of the remaining tiers will include six nodes of type $\beta$.
Case 3: nodes of type $\gamma$

For nodes of type $\gamma$, we find that $\mathbb{Q}_{i}$ is comprised of three of the six neighboring nodes, that is $\left|\mathbb{Q}_{i}\right|=3$. The three remaining nodes are not elements of $\mathbb{L}_{i}$ (they were already considered in the computation), and are consequently not elements in $\mathbb{Q}_{i}$. There are 5 link dependencies as denoted by the dashes in Figure 3.

\section{Case 4: node $L$}

Lastly, we note that we need to find $P\left(n_{L}\right)$ in order to solve (5). However, no dependencies need to be considered since we do not need to find node $L$ 's marginal probability.

For a triangular lattice topology whose number of tiers, $T$, is greater than 1 , there are exactly the following occurances of the above node types:

- node 1: 1 occurrence;

- node type $\beta$ : $6 T-1$ occurrences;

- node type $\gamma: \sum_{t=1}^{T} 6(t-1)=3 T(T-1)$ occurrences;

- node $L: 1$ occurance.

Hence, we re-write (5) in terms of nodes $1, \beta, \gamma$, and $L$ :

$$
\begin{aligned}
& P\left(n_{1}, n_{2}, \ldots, n_{L}\right)= \\
& \quad P\left(n_{1} \mid n_{2}, \ldots, n_{7}\right) \cdot P\left(n_{\beta} \mid n_{q_{1}^{\beta}}, n_{q_{2}^{\beta}}, n_{q_{3}^{\beta}}, n_{q_{4}^{\beta}}\right)^{6 T-1} . \\
& \quad P\left(n_{\gamma} \mid n_{q_{1}^{\gamma}}, n_{q_{2}^{\gamma}}, n_{q_{3}^{\gamma}}\right)^{3 T(T-1)} \cdot P\left(n_{L}\right)
\end{aligned}
$$

Finally substituting (6) into each of the conditional probabilities in (7) yield:

$$
\begin{aligned}
& P\left(n_{1}, n_{2}, \ldots, n_{L}\right)= \\
& \quad\left(\frac{P\left(n_{1}, n_{2}, \ldots, n_{7}\right)}{P\left(n_{2}, \ldots, n_{7}\right)}\right) \cdot\left(\frac{P\left(n_{\beta}, n_{q_{1}^{\beta}}, n_{q_{2}^{\beta}}, n_{q_{3}^{\beta}}, n_{q_{4}^{\beta}}\right)}{P\left(n_{q_{1}^{\beta}}, n_{q_{2}^{\beta}}, n_{q_{3}^{\beta}}, n_{q_{4}^{\beta}}\right)}\right)^{6 T-1} \cdot \\
& \quad\left(\frac{P\left(n_{\gamma}, n_{q_{1}^{\gamma}}, n_{q_{2}^{\gamma}}, n_{q_{3}^{\gamma}}\right)}{P\left(n_{q_{1}^{\gamma}}, n_{q_{2}^{\gamma}}, n_{q_{3}^{\gamma}}\right)}\right)^{3 T(T-1)} \cdot P\left(n_{L}\right)
\end{aligned}
$$

\section{B. Modeling the Finite Network Boundary}

We now extend our developed model to take into account the effect of a finite network. In such network, nodes belonging to the outer tier (i.e. nodes at the border of the network) will have reduced connectivity due to the reduced number of neighbors. Hence, two additional node types, $\lambda$ and $\lambda^{\circ}$, will need to be considered in the computation of the joint probability in (1). Figure 4 shows the locations of the various node types in triangular lattice topology whose $T=3$.

Case 5: nodes of type $\lambda$ and $\lambda^{\circ}$

We shall now consider nodes comprising the outer tier of a finite triangular lattice (i.e. nodes on the network boundary). We find that these nodes can only have either three neighbors (i.e. nodes $\lambda^{\circ}$ with $\left|\mathbb{D}_{i}\right|=3$ ) or four neighbors (i.e. nodes $\lambda$ with $\left.\left|\mathbb{D}_{i}\right|=4\right)$. Out of these three or four neighbors, only one (the node that follows in the same tier) remains an element in $\mathbb{L}_{i}$, the rest were already considered in the computation. Hence, $\left|\mathbb{Q}_{i}\right|=1$ for nodes of type $\lambda$ and $\lambda^{\circ}$. Therefore, there is 1 link dependency as denoted by the dash in Figure 5. 


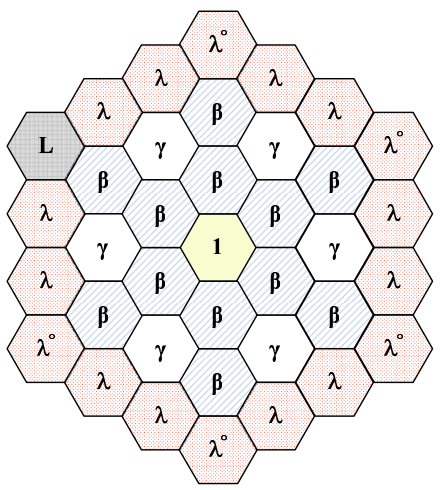

Fig. 4: Location of various node types in the finite topology
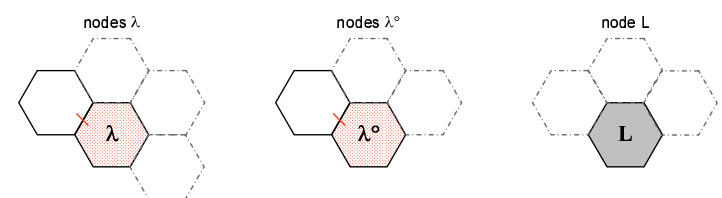

Fig. 5: Neighbors and Dependencies for additional node types

Note than node $L$ will only have 3 neighbors (i.e. $\left.\left|D_{i}\right|=3\right)$. However, no dependencies need to be considered since we do not need to find node L's marginal probability.

For a finite triangular lattice topology of $T>1$ tiers, there are exactly the following occurrences of the various node types (and combinations), each of which will have a different marginal probability:

- node 1: 1 occurrence;

- node type $\beta: 6(T-1)$ occurrences;

- node type $\gamma: \sum_{t=2}^{T-1} 6(t-1)=3 T^{2}-9 T+6$ occurrences;

- node type $\lambda$ followed by $\lambda: 6(T-2)$ occurrences;

- node type $\lambda$ followed by $\lambda^{\circ}$ (or $L$ ): 6 occurrences;

- node type $\lambda^{\circ}$ followed by $\lambda: 5$ occurrences.

- node $L: 1$ occurrence.

Hence, we re-write (5) in terms of nodes $1, \beta, \gamma, \lambda^{\circ}, \lambda$, and $L$ :

$$
\begin{aligned}
& P\left(n_{1}, n_{2}, \ldots, n_{L}\right)= \\
& P\left(n_{1} \mid n_{2}, \ldots, n_{7}\right), \cdot \\
& P\left(n_{\beta} \mid n_{q_{1}^{\beta}}, n_{q_{2}^{\beta}}, n_{q_{3}^{\beta}}, n_{q_{4}^{\beta}}\right)^{6(T-1)} . \\
& P\left(n_{\gamma} \mid n_{q_{1}^{\gamma}}, n_{q_{2}^{\gamma}}, n_{q_{3}^{\gamma}}\right)^{3 T^{2}-9 T+6} \cdot \\
& P\left(n_{\lambda} \mid n_{q_{1}^{\lambda}}\right)^{6(T-2)} \cdot P\left(n_{\lambda} \mid n_{q_{1}^{\lambda^{\circ}}}\right)^{6} \cdot P\left(n_{\lambda^{\circ}} \mid n_{q_{1}^{\lambda}}\right)^{5} . \\
& P\left(n_{L}\right)
\end{aligned}
$$

Finally substituting (6) into each of the conditional proba- bilities in (9) yield:

$$
\begin{aligned}
& P\left(n_{1}, n_{2}, \ldots, n_{L}\right)= \\
& \left(\frac{P\left(n_{1}, n_{2}, \ldots, n_{7}\right)}{P\left(n_{2}, \ldots, n_{7}\right)}\right) \text {. } \\
& \left(\frac{P\left(n_{\beta}, n_{q_{1}^{\beta}}, n_{q_{2}^{\beta}}, n_{q_{3}^{\beta}}, n_{q_{4}^{\beta}}\right)}{P\left(n_{q_{1}^{\beta}}, n_{q_{2}^{\beta}}, n_{q_{3}^{\beta}}, n_{q_{4}^{\beta}}\right)}\right)^{6(T-1)} . \\
& \left(\frac{P\left(n_{\gamma}, n_{q_{1}^{\gamma}}, n_{q_{2}^{\gamma}}, n_{q_{3}^{\gamma}}\right)}{P\left(n_{q_{1}^{\gamma}}, n_{q_{2}^{\gamma}}, n_{q_{3}^{\gamma}}\right)}\right)^{3 T^{2}-9 T+6} . \\
& \left(\frac{P\left(n_{\lambda}, n_{q_{1}^{\lambda}}\right)}{P\left(n_{q_{1}^{\lambda}}\right)}\right)^{6(T-2)} \cdot\left(\frac{P\left(n_{\lambda}, n_{q_{1}^{\lambda^{\circ}}}\right)}{P\left(n_{q_{1}^{\lambda^{\circ}}}\right)}\right)^{6} . \\
& \left(\frac{P\left(n_{\lambda^{\circ}}, n_{q_{1}^{\lambda}}\right)}{P\left(n_{q_{1}^{\lambda}}\right)}\right)^{5} \cdot P\left(n_{L}\right)
\end{aligned}
$$

\section{Computing the Model}

The computational complexity for computing the joint probability grows exponentially with the number of nodes in the network; the computational complexity in calculating $P\left(n_{i}, \ldots, n_{j}\right)$ is $O\left(c^{j-i+1}\right)$, where the constant $c$ represents the number of events preventing a node in a given topology from becoming isolated. Also note that $c$ grows exponentially with the topology, that is $c=2^{d_{\mathrm{T}}}-1$ where $d_{\mathbf{T}}=$ degree of a node in topology $\mathbf{T}\left(d_{\mathbf{T}}=6\right.$ for a triangular lattice topology).

The manual computation of (8) and (10) are quiet cumbersome. Hence, we developed a computer program to automate this process. We utilized a cluster of processors running on Sharcnet [8] in order to speed up the computation.

Due to space limitation and the complexity of the resulting computation, we have only included the closed form expressions of the constituents of the first term in (10). Refer to [9] and [10] for the rest of the computations.

$$
\begin{aligned}
& P\left(n_{1}, n_{2}, \ldots, n_{7}\right)= \\
& 87 r^{4} q^{26}+3678 r^{5} q^{25}+52913 r^{6} q^{24}+395652 r^{7} q^{23}+ \\
& 1906956 r^{8} q^{22}+6632116 r^{9} q^{21}+17782062 r^{10} q^{20}+ \\
& 38333088 r^{11} q^{19}+68331612 r^{12} q^{18}+102688686 r^{13} q^{17}+ \\
& 131860854 r^{14} q^{16}+146018842 r^{15} q^{15}+140287383 r^{16} q^{14}+ \\
& 117339336 r^{17} q^{13}+85551290 r^{18} q^{12}+54329784 r^{19} q^{11}+ \\
& 29970633 r^{20} q^{10}+14292982 r^{21} q^{9}+5850993 r^{22} q^{8}+ \\
& 2035632 r^{23} q^{7}+593768 r^{24} q^{6}+142506 r^{25} q^{5}+ \\
& 27405 r^{26} q^{4}+4060 r^{27} q^{3}+435 r^{28} q^{2}+30 r^{29} q+r^{30}
\end{aligned}
$$

where $q=1-r$, and 


$$
\begin{aligned}
& P\left(n_{2}, \ldots, n_{7}\right)= \\
& 2 r^{3} q^{27}+249 r^{4} q^{26}+6582 r^{5} q^{25}+76365 r^{6} q^{24}+ \\
& 505440 r^{7} q^{23}+2253024 r^{8} q^{22}+7432948 r^{9} q^{21}+ \\
& 19215999 r^{10} q^{20}+40387200 r^{11} q^{19}+ \\
& \quad 70736989 r^{12} q^{18}+105022794 r^{13} q^{17}+ \\
& 133752441 r^{14} q^{16}+147303096 r^{15} q^{15}+ \\
& 141017040 r^{16} q^{14}+117684414 r^{17} q^{13}+ \\
& \quad 85685772 r^{18} q^{12}+54372282 r^{19} q^{11}+ \\
& 29981259 r^{20} q^{10}+14295006 r^{21} q^{9}+5851269 r^{22} q^{8}+ \\
& 2035656 r^{23} q^{7}+593769 r^{24} q^{6}+142506 r^{25} q^{5}+ \\
& 27405 r^{26} q^{4}+4060 r^{27} q^{3}+435 r^{28} q^{2}+30 r^{29} q+r^{30}
\end{aligned}
$$

While understanding the resulting computations in (11) and (12) is cumbersome, we will give few brief insights. For simplicity, consider Equation (12). We note that the last term resembles the probability of one extreme event occurring, that is when all 36 links ( 6 links/node $* 6$ nodes) are connected. Since 6 out of the 36 inter-node links are common (i.e. dependant), the probability of such event occurring is $r^{30}$. Similarly, the first resembles the probability of the other extreme event, when the 6 nodes are connected just enough for none of them to be isolated. This event occurs when only links between 3 pairs of adjacent nodes (i.e. nodes 2-3, 4-5, 6-7 or 7-2, 3-4, 5-6) are connected, the remaining 27 links are not connected. Since such event can occur in two different ways, the resulting probability is $2 r^{3} q^{27}$. The middle terms in (12) resemble the probabilities of all other events between the two discussed extremes.

\section{Results}

\section{A. Effect of Independent Links}

We present the probability that none of the nodes are isolated for networks composed of 1, 3, and 5 tiers found using our generated closed form solution that takes into account internode link dependency. We compare our results to computations based on the common assumption of internode link independence.

In assuming independence of links and nodes in a triangular lattice topology, (4) simplifies to:

$$
P(\text { no node isolated })^{*}=\left(1-(1-r)^{6}\right)^{1+3 T(T+1)}
$$

The simplification in the above equation is due to the fact that all nodes in the topology have 6 neighbors; the total number of nodes is $1+\sum_{t=1}^{T} 6 t=1+3 T(T+1)$ (we added 1 for the center node).

Figure 6a plots three sets of results corresponding to the three sized networks. For each set, we plot our closed form solution given by the computation resulting from (8), and its independent link counterpart given by (13). Few observations are noteworthy. Firstly, as expected, $P$ (no node isolated) increases with increasing probability of link connectivity, $r$. Furthermore, for a given value of $r, P$ (no node isolated $)$ decreases as the number of nodes in a network increase. This is expected since the addition of each node into the network enhances the chances of at least one node in the network becoming isolated. Lastly, the computed $P$ (no node isolated) exceeds the claimed upper bound $P(\text { no node isolated })^{*}$. Therefore, we proved that there are cases when the network connectivity can exceed $P(\text { no node isolated })^{*}$, and hence established a new upper bound that can be computed using (8).

Figure $6 \mathrm{~b}$ plots the percentage of error in $P($ no node isolated $)$ due to the assumption of independent links and nodes connectivities. It is evident that the error decreases with increasing values of $r$; it is almost negligible for $r \geq 0.6$. It is also evident that the error increases with increasing number of nodes. Hence, for a given network size, we can quantify the error resulting from the assumption of link and node independence, as well as identify the critical range of $r$ at which the error is unacceptable for a given application.

\section{B. Effect of Infinite Boundary}

Figure 7 highlights the effect of assuming an infinite boundary on the connectivity of a triangular lattice topology. We first plot in Figure 7a our closed form expression resulting from (10). In the same figure, the closed form expression resulting from our earlier model which takes into account the effect of link dependence but ignored the effect of an outer boundary is given for comparison. It is evident that $P$ (no node isolated $)$ is erroneously higher when an infinite boundary is assumed. This is contributed to the fact that outer tier nodes are only surrounded by three or four neighbors (verses six when an infinite boundary is assumed), therefore causing a significant reduction in connectivity.

Figure $7 \mathrm{~b}$ plots the percentage error in $P$ (no node isolated $)$ due to assuming an infinitely large topology. We observe that the error follows a bell-curve pattern; it first increases to reach a peak, then starts decreasing as the link connectivity increase. We also observe that the magnitude of the peak error increases dramatically with increasing network size; the peak error percentages are $543 \%, 8960 \%$, and $124000 \%$ for the 1 , 3 , and 5 tiers respectively. Moreover, the error only drops to $10 \%$ when $r \geq 0.75$ (this is not evident from the figure due to the big range on the y-axis). This hence shows that ignoring the effect of the network boundary has a non-negligible effect of overestimating the connectivity of the network.

\section{CONCLUSION}

In this work, we presented a novel methodology for finding a closed form upper bound for wireless mesh network connectivity. Our solution is the first to take into account the effect of both: interdependence of connectivity of spatially co-located nodes; and the effect of network boundary nodes' connectivity. We used the methodology to find the connectivity in the optimal triangular lattice topology. We first analyzed 


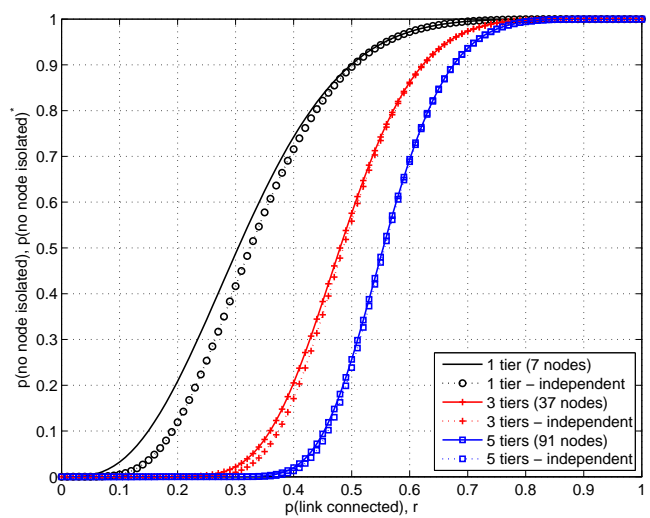

(a) Probability that none of the nodes are isolated

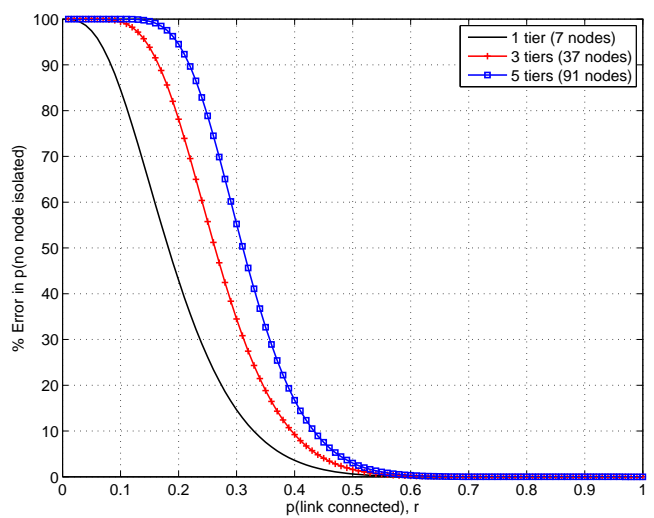

(b) Percentage error due to link independence

Fig. 6: Effect of assuming independent links on the connectivity of triangular lattice topologies

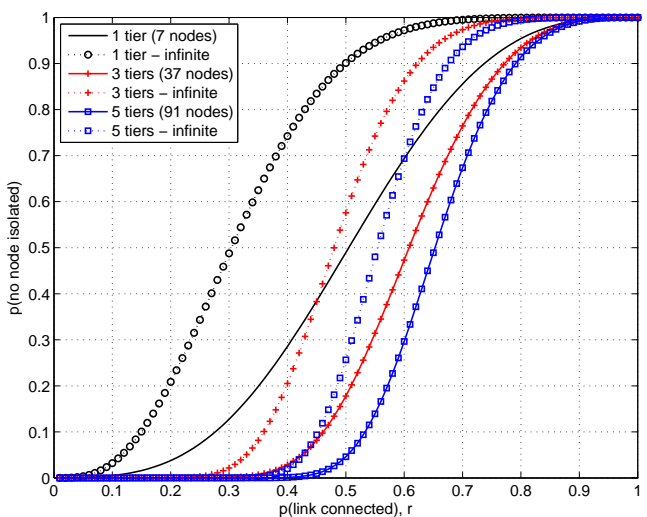

(a) Probability that none of the nodes are isolated

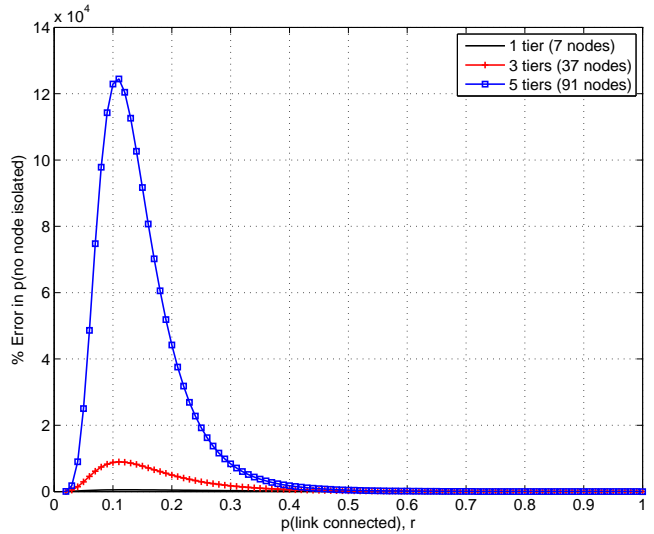

(b) Percentage error due to an infinite boundary

Fig. 7: Effect of assuming an infinite boundary on the connectivity of triangular lattice topologies

the impact and validity of the commonly utilized assumption of link independence. Results obtained show that there are cases where the connectivity exceeds $P$ (no node isolated $)^{*}$ that assumes independent links between nodes. Hence, the assumption of independent links between co-located nodes is invalid, and our derived expression for connectivity serve as a new upper bound for network connectivity. We have also examined the impact that the assumption of infinite network boundary has on the network connectivity. Results show that for most cases, this assumption overestimates the network connectivity by a significant amount, and is hence invalid. Using the proposed methodology, we quantified the errors resulting from the two commonly used assumptions for triangular lattice networks. We have also shown that the magnitudes of the percentage error resulting from either assumptions increase with the size of the network. The developed methodology can similarly be applied to any network topology with equal distance node allocation. The qualitative results obtained will generally hold for similar topologies.

\section{REFERENCES}

[1] C. Bettstetter and C. Hartmann, "Connectivity of Wireless Multihop Networks in a Shadow Fading Environment," Wireless Networks, vol. 11, no. 5, pp. 571-579, 2005.

[2] P. Stuedi, O. Chinellato, and G. Alonso, "Connectivity in the presence of shadowing in 802.11 ad hoc networks," Wireless Communications and Networking Conference, 2005 IEEE, vol. 4, 2005.

[3] O. Dousse, P. Thiran, and M. Hasler, "Connectivity in ad-hoc and hybrid networks," INFOCOM 2002. Twenty-First Annual Joint Conference of the IEEE Computer and Communications Societies. Proceedings. IEEE, vol. 2, 2002.

[4] J. Camp, J. Robinson, C. Steger, and E. Knightly, "Measurement driven deployment of a two-tier urban mesh access network," in MobiSys 2006 : Proceedings of the 4th international conference on Mobile systems, applications and services. New York, NY, USA: ACM Press, 2006, pp. 96-109.

[5] D. Chen, M. Haenggi, and J. N. Laneman, "Distributed spectrumefficient routing algorithms in wireless networks," in Proc. Conf. Inform. Sci. and Syst. (CISS), Mar 2007.

[6] P. Erdös and A. Rényi, "On the evolution of random graphs," Bulletin of the Institute of International Statistics, vol. 38, pp. 343-347, 1961.

[7] R. Kershner, "The Number of Circles Covering a Set," American Journal of Mathematics, vol. 61, no. 3, pp. 665-671, 1939.

[8] "Shared hierarchical academic research computing network," www. sharcnet.ca.

[9] M. Abou El Saoud, "Connectivity in Wireless Mesh Networks," Systems and Computer Engineering, Carleton University, Tech. Rep. SCE-07-07, Aug 2007.

[10] M. Abou El Saoud and Samy Mahmoud, "Effect of a Finite Boundary on the Connectivity of Triangular Lattice Topologies," Systems and Computer Engineering, Carleton University, Tech. Rep. SCE-07-08, Aug 2007. 